\title{
Controle de um Sistema de Navegação de um Robô Ambiental Híbrido por meio de um Sistema de Inferência Fuzzy Hierárquico
}

\author{
C.J. GÓMEZ, M. VELLASCO* e R. TANSCHEIT \\ Recebido em 4 de maio de 2017 / Aceito em 5 de dezembro de 2017
}

\begin{abstract}
RESUMO. Apresenta-se, neste artigo, o desenvolvimento completo de um sistema de navegação auxiliar baseado na Lógica Fuzzy para um robô móvel denominado Robô Ambiental Híbrido Médio (RAHM). Apresenta-se também o projeto eletrônico e a programação do sistema de navegação. O desempenho deste sistema é avaliado frente ao do Sistema Principal de Navegação do RAHM.
\end{abstract}

Palavras-chave: Lógica Fuzzy; Navegação; Robótica autônoma; Controle.

\section{INTRODUÇÃO}

A exploração de óleo e gás na floresta amazônica teve como um de seus resultados a construção de um gasoduto ao longo do rio Solimões, ligando as cidades de Coari a Manaus. Devido ao impacto que tal atividade provoca na natureza, as atividades de produção e transporte de óleo e gás na região e seus potenciais impactos socioambientais merecem constante acompanhamento. Pesquisadores e técnicos percorrem o Rio Solimões trimestralmente para colher amostras e dados sobre o ecossistema e as populações humanas. No entanto, há uma série de dificuldades para a coleta de tais amostras, como a constante presença de animais selvagens e insetos transmissores de doenças, além da inacessibilidade de certos lugares como regiões de plantas flutuantes [12].

A indústria brasileira tem desenvolvido novas abordagens para as tarefas de monitoramento e inspeção de gasodutos. Para possibilitar a coleta de informações e amostras de uma maneira segura nas áreas remotas, bem como para estabelecer pontos móveis de monitoramento ambiental, foi desenvolvido pelo Centro de Pesquisas da Petrobras (CENPES) o Robô Ambiental Híbrido (RAH) [12]. Este robô móvel, dotado de equipamentos para a coleta de informação, deveria satisfazer os requisitos exigidos pelo ambiente amazônico, com capacidade de se movimentar em terra firme, pântano, lama, areia, brejos e terrenos irregulares. Os testes realizados demonstraram que o RAH (Figura 1), dotado de um sistema de navegação, foi capaz de atender aos requisitos acima [2], podendo ser empregado na coleta de amostras da fauna e flora local, em medições

\footnotetext{
*Autor correspondente: M. Vellasco - E-mail: marley@ele.puc-rio.br

Departamento de Engenharia Elétrica, PUC-Rio, Rua Marquês de S. Vicente, 225 22451-900 Rio de Janeiro, RJ, Brasil.

E-mail: cristhian.julian@ hotmail.com; marley@ele.puc-rio.br; ricardo@ele.puc-rio.br
} 
de parâmetros físico-químicos do meio e ainda na detecção de níveis de gás metano em regiões alagadas como decorrência da construção de usinas hidroelétricas.

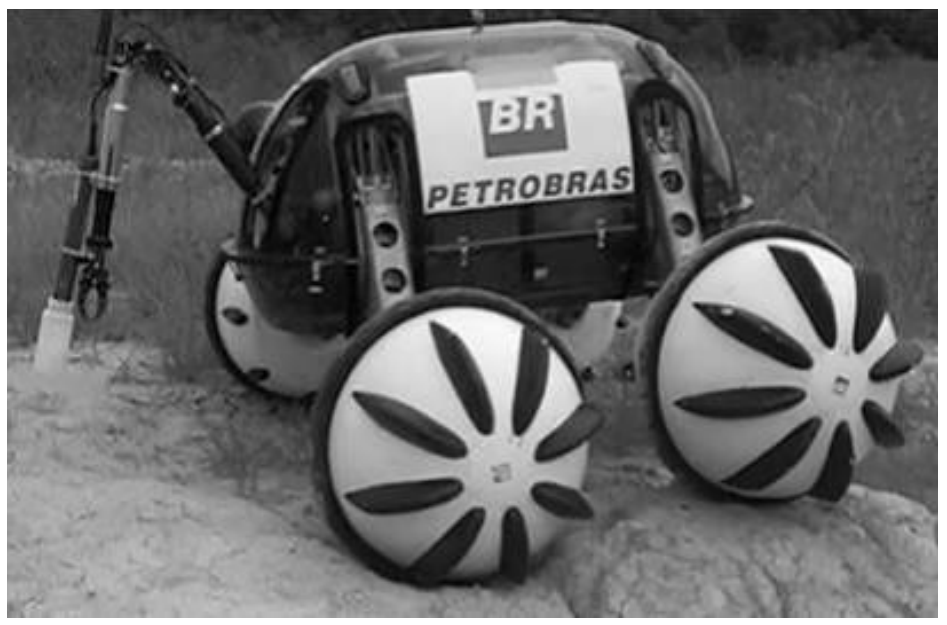

Figura 1: Protótipo RAH 2011. Fonte Relatório Ativatec.

Aprimoramentos nas partes mecânica e eletrônica e na capacidade de navegação autônoma deram origem a uma nova versão do RAH, denominada Robô Ambiental Híbrido Médio (RAHM) (Figura 2) [5], destinado a operar na região do gasoduto Coari-Manaus. A palavra Médio indica que o RAHM é um pouco menor do que o RAH. Exige-se deste robô a capacidade de se desviar de obstáculos e de detectar rotas mais adequadas para atingir objetivos estabelecidos.

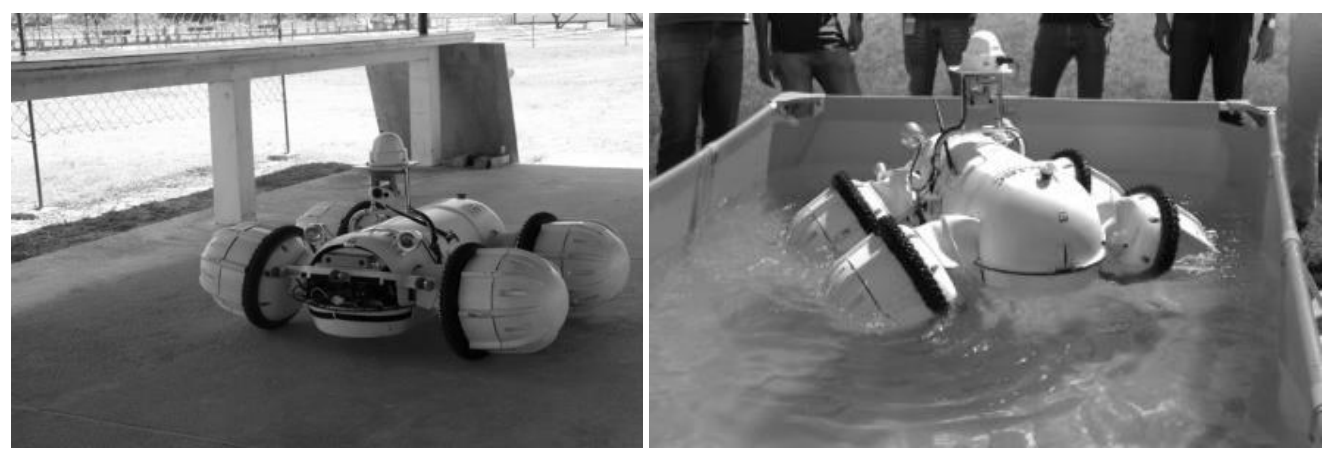

Figura 2: Versão atual do RAHM.

O RAHM é dotado de um Sistema Primário de Navegação (SPN) e necessitava de um Sistema Auxiliar de Navegação (SAN) para atuação em caso de falha. Considerando que as técnicas de inteligência computacional [7], especialmente a Lógica Fuzzy [6] [8], têm apresentado bom desempenho em situações práticas [13] [16], inclusive no campo da robótica móvel [11] [10] [9], optou-se por construir o Sistema de Navegação Auxiliar com o apoio desta técnica. Este trabalho 
resume o desenvolvimento deste sistema, extensivamente abordado em [5] - para operar em caso de falha do SPN -, incluindo o projeto da eletrônica e do Sistema de inferência Fuzzy. O desempenho do Sistema Auxiliar de Navegação é comparado ao do Sistema Primário, inclusive no que diz respeito à relação custo-benefício de cada um deles.

\section{CONTROLE DO RAHM}

Conforme mencionado, o RAHM é um protótipo robótico cujo objetivo principal é o monitoramento do entorno do gasoduto Coari-Manaus. O RAHM possui três tipos de funcionamento: automático, teleoperado e semiautomático.

No modo automático, o RAHM é completamente autônomo e baseia a sua navegação em equipamentos sofisticados - sensores a laser e câmeras -, que fornecem informações para o Sistema Primário de Navegação (SPN). Este faz uso de um computador remoto e de um computador embarcado para processar os dados e definir os sinais que serão enviados aos atuadores.

No modo de teleoperação, o RAHM responde somente à tração, câmera IP e canal de comunicação, ou seja, os equipamentos vitais para que o operador possa movimentar o robô até atingir uma zona segura para inspeção e reparação.

O modo semiautomático é uma opção para situações de falha ou de emergência que afetem a operação do SPN. Equipamentos são desligados e a navegação se dá por meio de instrumentos mais simples, com o objetivo de poupar energia e recursos computacionais. O Sistema Auxiliar de Navegação (SAN), descrito a seguir, foi desenvolvido para atuar exatamente nestas circunstâncias, de forma a assegurar um funcionamento mínimo mas ainda adequado.

\section{SISTEMA AUXILIAR DE NAVEGAÇÃO}

No desenvolvimento do SAN, estabeleceu-se que ele deveria apresentar consumo de energia mínimo, custo financeiro e computacional baixo e simplicidade no cumprimento do objetivo geral. Estes objetivos nortearam as escolhas dos equipamentos (placa Arduino e sensores de ultrassom, por exemplo). O desenvolvimento compreendeu o projeto eletrônico, seleção de equipamentos, programação, enlaces de comunicação, entre outras tarefas, conforme detalhado em [5]. Os cinco módulos do SAN são apresentados na Figura 3: Módulo de Sensores, Módulo Processador, Módulo de Comunicação, Módulo PC Remoto e Módulo de Atuação.

O módulo de sensores é encarregado de adquirir a informação do entorno do robô e o processador (placa Arduino MEGA) tem a função de codificar e interpretar essa informação. O módulo de comunicação é responsável pela troca de informação, via radiofrequência, entre o robô e o computador remoto, onde o SIF está implementado. Este módulo compreende o switch e os rádios transmissor e receptor. Os atuadores têm a função de locomover o robô de acordo com as saídas fornecidas pelo SIF.

A definição do número de sensores e sua localização foi resultado de uma série de testes com diversas configurações. Várias possibilidades foram consideradas, levando também em conta 


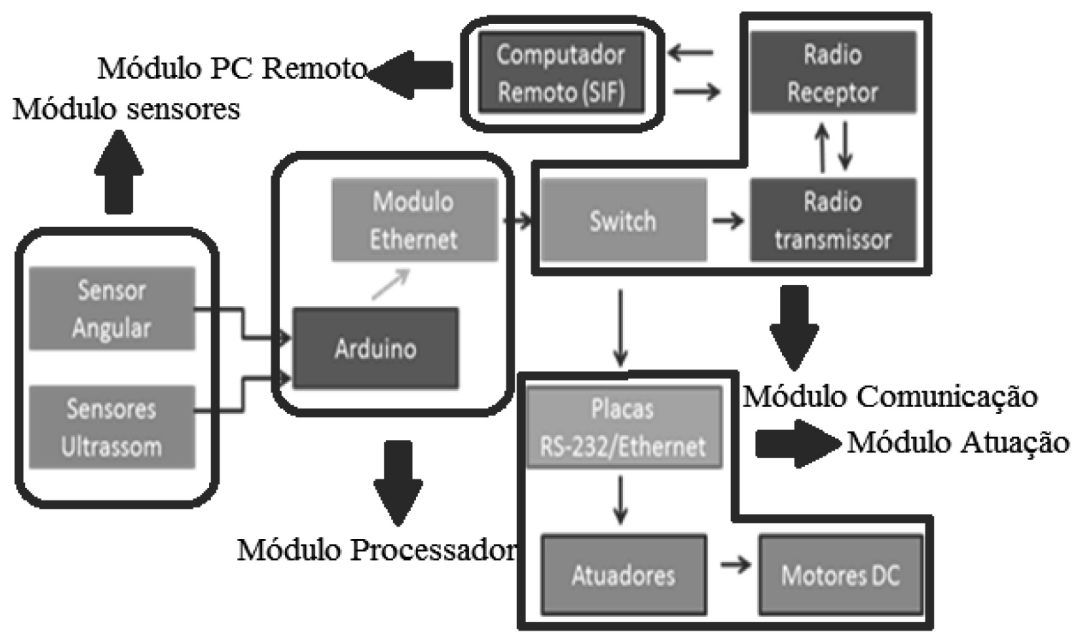

Figura 3: Módulos do Sistema Auxiliar de Navegação.

trabalhos disponíveis na literatura [4] [15] [3]. A disposição final dos sensores é apresentada na Figura 4, onde se observam os cinco sensores de ultrassom: três na parte frontal do robô - área mais importante em se tratando de navegação -, um na lateral esquerda e um na lateral direita. Há também um sensor angular, localizado na parte central do robô.

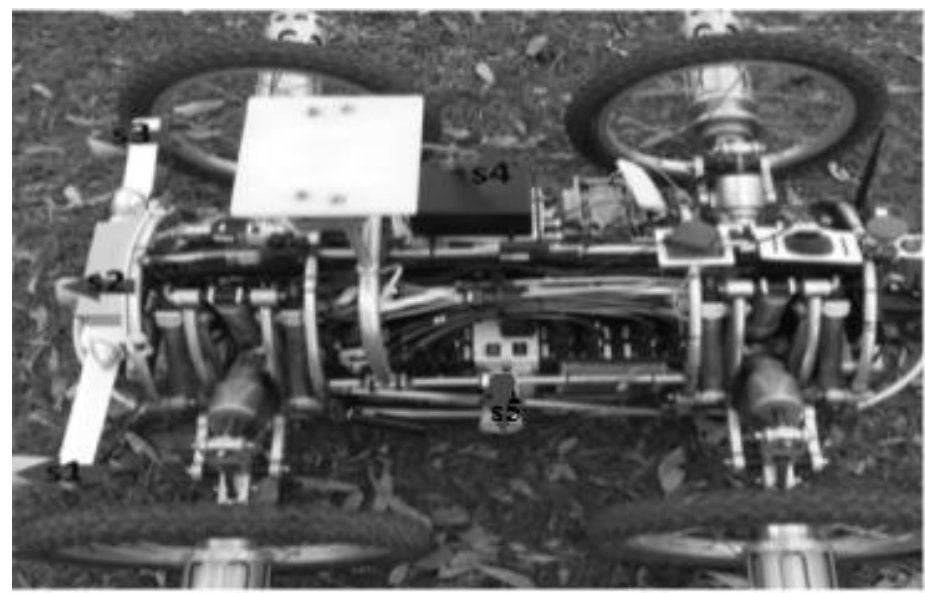

Figura 4: Distribuição dos sensores de ultrassom.

\subsection{Sistema de Inferência Fuzzy Geral}

Considerando o número de variáveis - seis sinais provindos dos sensores - necessárias para o controle do robô no modo semiautomático, optou-se pelo desenvolvimento de um SIF 
hierárquico, reduzindo-se, assim, o número total de regras. Um menor número de regras não apresenta vantagem significativa do ponto de vista operacional, mas torna mais simples a tarefa dos especialistas na construção das bases de regras e também favorece a interpretabilidade. Assim, o SIF Geral é composto por dois subsistemas de inferência fuzzy: o Sistema Intermediário (SIF1), identificado na Figura 5 pelo módulo "Área Frontal", e o Sistema de Controle (SIF 2) propriamente dito, identificado pelo módulo "Sistema". O SIF1 obtém a informação dos obstáculos à frente do robô - distância para os obstáculos e a sua orientação em relação ao objetivo. O SIF 2 recebe a informação de saída do sistema intermediário e, juntamente com os sensores que detectam obstáculos nas zonas laterais, fornece os valores de saída para os atuadores. Observe-se que o SIF Geral possui as seis entradas acima referidas: sinais de cinco sensores de ultrassom e de um sensor angular.

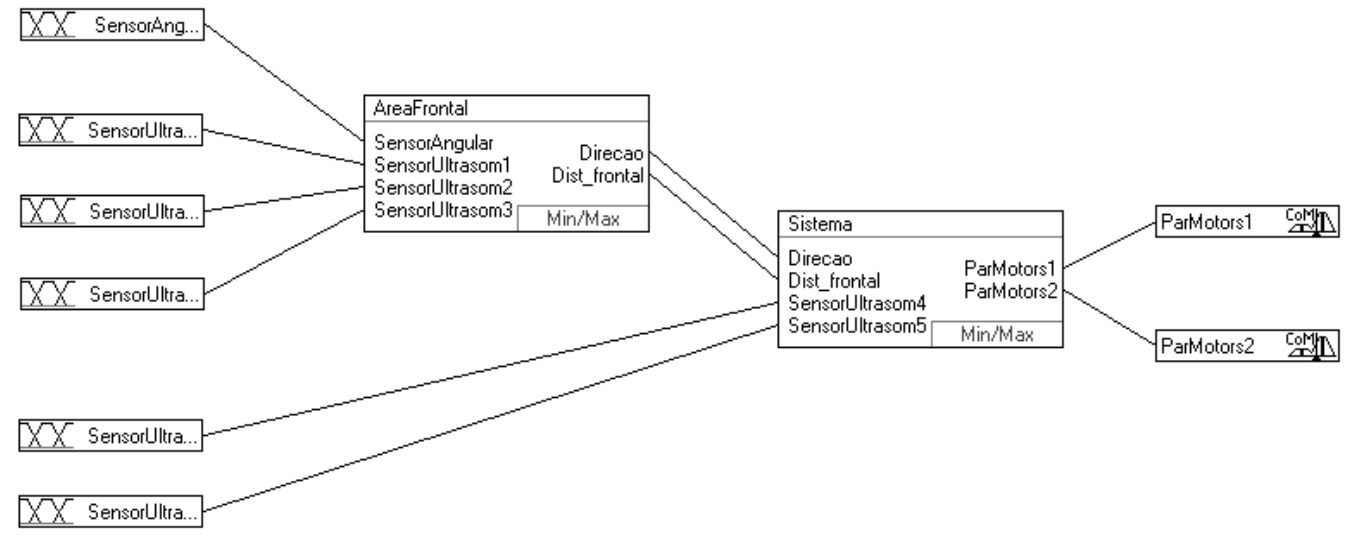

Figura 5: Sistema de Inferência Fuzzy Geral.

O comportamento do robô é resultado da diferença de velocidade angular entre os pares de motores do lado esquerdo e os pares de motores do lado direito. São necessárias, portanto, as duas saídas mostradas na Figura 5. Uma saída envia o mesmo valor para os dois motores do lado esquerdo (PM1) e a outra envia a informação para os dois motores do lado direito (PM2). Assim, a trajetória a ser percorrida pelo robô é determinada pela diferença de velocidades angulares entre PM1 e PM2 (vide Figura 6).

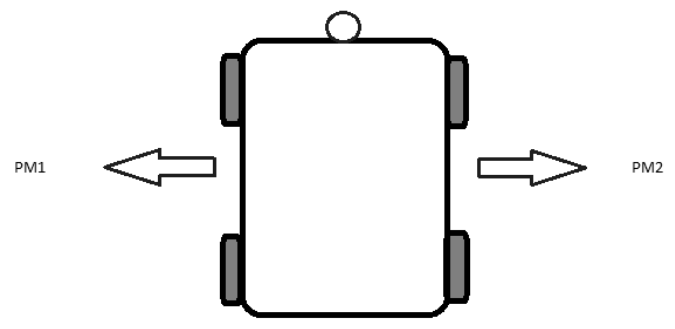

Figura 6: Pares de motores. 


\subsection{SIF1: Sistema Intermediário}

Como a informação referente à parte frontal é vital para o robô lograr se desviar de obstáculos, três dos cinco sensores de ultrassom são colocados nessa região. A informação fornecida por estes três sensores, juntamente com a do sensor angular, é responsável pela capacidade de o robô se desviar de obstáculos. A partir dos sinais recebidos destes quatro sensores, o Sistema Intermediário (Figura 7) fornece como saídas a orientação a ser seguida pelo robô e a distância média geral dos obstáculos na zona frontal.

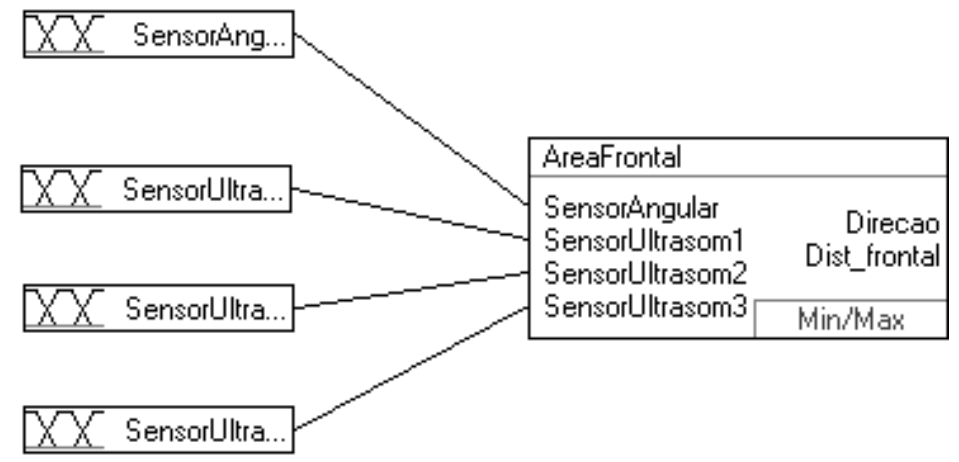

Figura 7: Sistema Intermediário.

As duas saídas intermediárias direção e distância frontal são defuzzificadas e utilizadas como entradas do SIF2 (Sistema de Controle).

\subsubsection{Variáveis de Entrada}

Os sensores de ultrassom oferecem a informação de distância de qualquer obstáculo que seja detectado em uma faixa de $30 \mathrm{~cm}$ a 5 metros na linha de visão do robô. O sensor angular fornece a orientação da frente do robô em relação ao objetivo, isto é, o ângulo de desvio em relação a um alvo previamente especificado. Apresentam-se a seguir os termos linguísticos (conforme nomenclatura utilizada em [5]) e os conjuntos fuzzy, definidos por funções de pertinência, correspondentes às variáveis de entrada consideradas.

- Distância ao Obstáculo (Ultrassom1, 2 e 3): as funções de pertinência para as três entradas do Sistema Intermediário (dados de três sensores) que dizem respeito à distância do obstáculo são mostradas na Figura 8, cabendo a elas os seguintes termos linguísticos: Close, Medium e Far.

- Ângulo de Desvio (SensorAngular): os cinco termos linguísticos (High_Left, Medium_Left, Center, Medium_Right, High_Right) e as funções pertinência correspondentes ao ângulo de desvio são mostradas na Figura 9. 


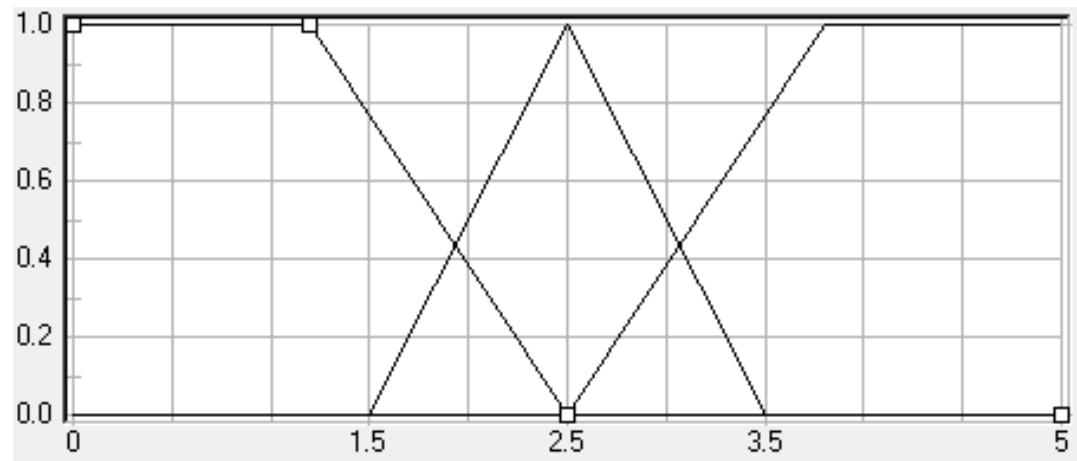

Figura 8: Distância ao Obstáculo.

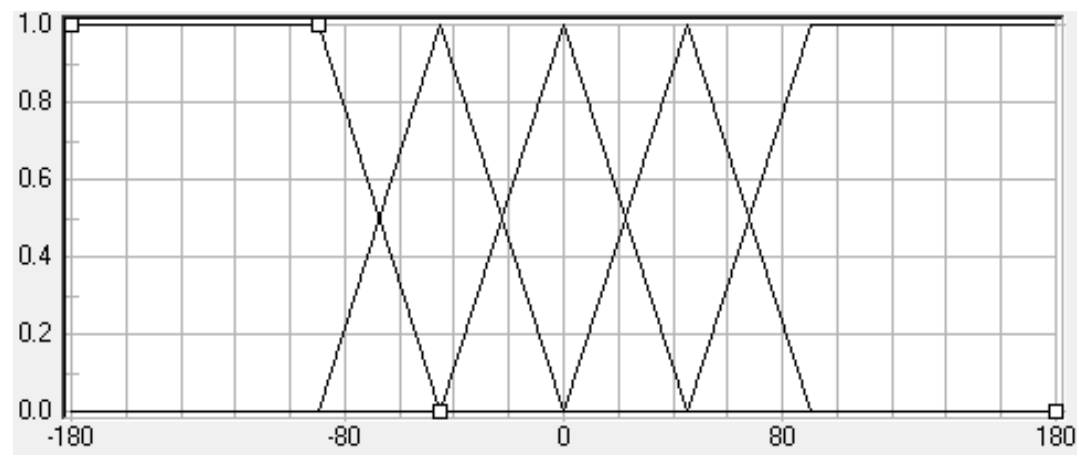

Figura 9: Ângulo de desvio.

\subsubsection{Variáveis de Saída}

As saídas do SIF1 são: distância média em relação ao obstáculo que se encontra à frente do robô (Distância Frontal) e ângulo de direção (Direção) que o robô deve seguir para manter a sua trajetória até a posição desejada.

Para a saída Distância Frontal, os termos linguísticos e funções de pertinência são idênticos aos utilizados para as entradas relativas à distância (Figura 8). Para a saída Direção, as funções de pertinência são iguais às mostradas na Figura 9 para a variável de entrada Ângulo de Desvio, com os termos linguísticos Very_Left, Left, Center, Right e Very_Right.

\subsubsection{Base de Regras}

Como o Sistema Intermediário (SIF1) recebe três informações de distância, com três termos linguísticos cada, e uma do sensor angular, com cinco termos linguísticos, o número de regras possíveis é 135. O conjunto completo de regras pode ser encontrado em um apêndice de [5]. Exemplos de regras são apresentados abaixo: 
IF (SensorAngular IS High_Left) AND (Ultrassom1 IS Medium) AND (Ultrassom2 IS Close) AND (Ultrassom3 IS Medium) THEN Dist_Frontal IS Close AND Direcao IS Very_Right

IF (SensorAngular IS Medium_Left) AND (Ultrassom1 IS Close) AND (Ultrassom2 IS Close) AND (Ultrassom3 IS Close) THEN Dist_Frontal IS Close AND Direcao IS Very_Right

IF (SensorAngular IS Center) AND (Ultrassom1 IS Far) AND (Ultrassom2 IS Close) AND (Ultrassom3 IS Close) THEN Dist_Frontal IS Close AND Direcao IS Left

IF (SensorAngular IS Medium_Right) AND (Ultrassom1 IS Medium) AND (Ultrassom2 IS Medium) AND (Ultrassom3 IS Medium) THEN Dist_Frontal IS Medium AND Direcao IS Left

IF (SensorAngular IS High_Right) AND (Ultrassom1 IS Far) AND (Ultrassom2 IS Far) AND (Ultrassom3 IS Far) THEN Dist_Frontal IS Far AND Direcao IS Very_Left

\subsection{SIF2: Sistema de Controle}

Este sistema (SIF2) recebe como entradas os valores defuzzificados do SIF1, além das informações dos sensores localizados nas zonas laterais do robô, e fornece na saída a informação para os pares de motores. A Figura 10 apresenta a estrutura do SIF2, com suas quatro entradas e duas saídas.

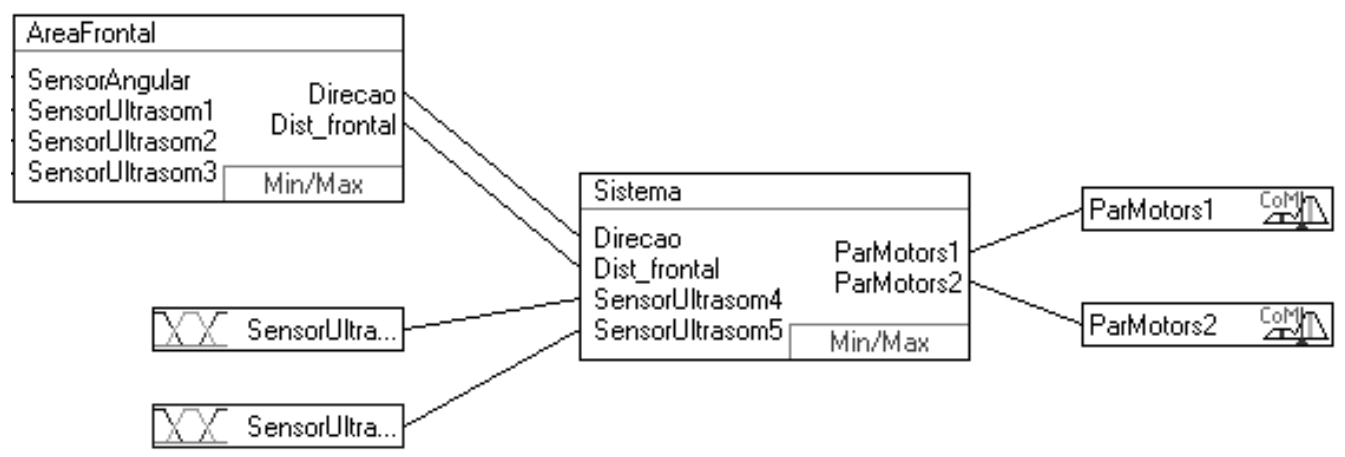

Figura 10: Sistema de Inferência 2 (Sistema de Controle).

\subsubsection{Variáveis de Entrada}

Como mencionado, são quatro as variáveis de entrada deste sistema:

- Distância Frontal e Direção: fornecidas pelo Sistema Intermediário, possuem as características apresentadas nas Figura 8 e 9, respectivamente. 
- Distâncias ao Obstáculo: fornecidas pelos sinais dos sensores de ultrassom localizados nas laterais direita (sensor ultrassom 4) e esquerda (sensor ultrassom 5), possuem as mesmas características dos sensores de ultrassom do Sistema intermediário.

\subsubsection{Variáveis de Saída}

Conforme mencionado, o SIF 2 fornece as duas saídas que serão utilizadas para acionar o par de motores ilustrado na Figura 6. Valores de tensão proporcionais ao valores resultantes do processo de inferência - entre -5000 rpm e $5000 \mathrm{rpm}$ - são fornecidos aos motores de tração. O sinal positivo ou negativo informa o sentido de rotação do motor, que corresponde ao movimento para frente ou para trás do robô. A velocidade máxima do robô é de $1 \mathrm{~m} / \mathrm{s}$.

Na Figura 11 são apresentadas as funções de pertinência correspondentes às variáveis de saída do SIF 2, distribuídas em um universo de discurso abrangendo valores entre -5000 e +5000 . Os termos linguísticos a elas associados são: Rever, Slow, Medium e Fast. A distribuição assimétrica das funções de pertinência deve-se à necessidade de se ter um controle mais fino quando o robô se movimenta para a frente. Quando há um bloqueio total à frente e nas laterais, o robô deve se deslocar para trás (Rever) até atingir uma região mais livre. Neste caso, o controle não necessita ser tão fino.

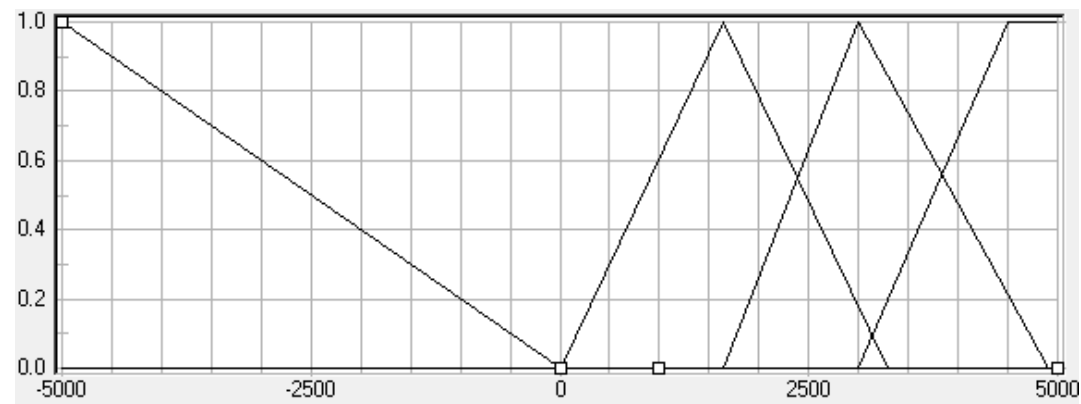

Figura 11: Variáveis de saída.

\subsubsection{Base de Regras}

A exemplo do SIF Intermediário, o número de regras possíveis é 135 (disponíveis em [5]); a seguir apresentam-se alguns exemplos de regras:

IF (Direcao IS Very_Left) AND (Dist_Frontal IS Far) AND (Ultrassom4 IS Medium) AND (Ultrassom5 IS Medium) THEN PM1 IS Medium AND PM2 IS Fast

IF (Direcao IS Left) AND (Dist_Frontal IS Close) AND (Ultrassom4 IS Close) AND (Ultrassom5 IS Medium) THEN PM1 IS Slow AND PM2 IS Fast 
IF (Direcao IS Center) AND (Dist_Frontal IS Close) AND (Ultrassom4 IS Close) AND (Ultrassom5 IS Close) THEN PM1 IS Rever AND PM2 IS Rever

IF (Direcao IS Right) AND (Dist_Frontal IS Close) AND (Ultrassom4 IS Medium) AND (Ultrassom5 IS Close) THEN PM1 IS Fast AND PM2 IS Slow

IF (Direcao IS Very_Right) AND (Dist_Frontal IS Far) AND (Ultrassom4 IS Close) AND (Ultrassom5 IS Medium) THEN PM1 IS Medium AND PM2 IS Fast

\subsubsection{Defuzzificação}

Para os dois SIFs, utilizou-se o método de centro dos máximos [14].

\subsection{Implementação do SIF Geral}

O SIF hierárquico apresentado nas seções anteriores foi programado em uma plataforma que permitia a sua conexão com os dispositivos existentes no projeto eletrônico. A ferramenta Visual Studio [1] foi a escolhida, pois apresenta os seguintes requisitos fundamentais para a programação do SIF:

- Possui componente para receber dados através da porta ethernet, já que os dados obtidos pelos sensores são enviados por radio frequência ao PC remoto, o qual os recebe pela porta ethernet.

- Possui componente para enviar dados através da porta serial, pois as ordens de controle dadas pelo controlador fuzzy são enviadas aos atuadores das rodas através da placa conversora RS232/Ethernet pela porta serial.

- Permite o desenvolvimento de uma interface gráfica para o monitoramento de todas as variáveis de entrada e saída.

- Possui biblioteca de Lógica Fuzzy na ferramenta.

Desenvolveu-se uma interface, conforme mostrado na Figura 12, para realizar o monitoramento de todas as informações do robô em tempo real. A parte esquerda da interface apresenta uma imagem do RAHM, com a distribuição dos cinco sensores de ultrassom (três frontais e dois laterais) e seus respectivos valores. Na parte inferior do lado esquerdo, pode-se visualizar o dado recebido do sensor angular. Na parte central, visualizam-se os dois valores de saída do Sistema Intermediário: direção e a distância média da zona frontal.

Tem-se, à direita, outra imagem do robô, onde são monitorados os dados enviados para os quatro atuadores das rodas de tração. A localização de cada caixa corresponde à localização real do atuador que controla a roda. 


\section{Sistema SIF para o RAHM}

\section{Entradas}

\section{Sensores de Ultrassom}

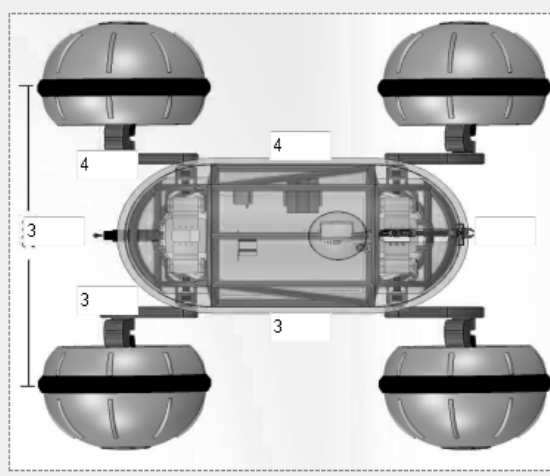

Saidas
Angulo Direcao

Sensor Angular

Figura 12: Interface Gráfica de monitoramento.

\section{EXPERIMENTOS E RESULTADOS}

Para avaliar o comportamento do Sistema de Navegação Auxiliar frente ao do Sistema de Navegação Primário, foram criados dez cenários controlados (distribuições de obstáculos). Esta comparação envolveu, além das trajetórias percorridas, itens como custo financeiro, custo computacional, consumo de energia, tempo de percurso e fluxo de dados. Recorde-se que o projeto do SAN pretendia aliar um desempenho satisfatório com custo baixo.

Os dez experimentos foram realizados em um mesmo ambiente plano, de 50m x 30m, com diferentes configurações de obstáculos. O robô deveria completar o trajeto até o ponto final especificado, esquivando-se de todos os obstáculos existentes.

\subsection{Testes em Ambientes Controlados}

Esta seção apresenta os dez experimentos realizados para avaliar o Sistema de Navegação Auxiliar. Tais experimentos são ilustrados nas imagens a seguir, extraídas de vídeos, em que se apresentam os trajetos do robô utilizando o SPN (linha contínua) e o SAN (linha pontilhada). 
- Experimento 1: Conforme ilustrado na Figura 13, este experimento considera três obstáculos distribuídos de forma que seja indiferente a escolha, pelo robô, da rota a seguir (pelo lado esquerdo ou direito). O objetivo encontra-se a 50 metros de distância. $\mathrm{O}$ robô, quando controlado pelo SAN, logrou chegar ao objetivo, mas sem percorrer a rota mais eficiente.

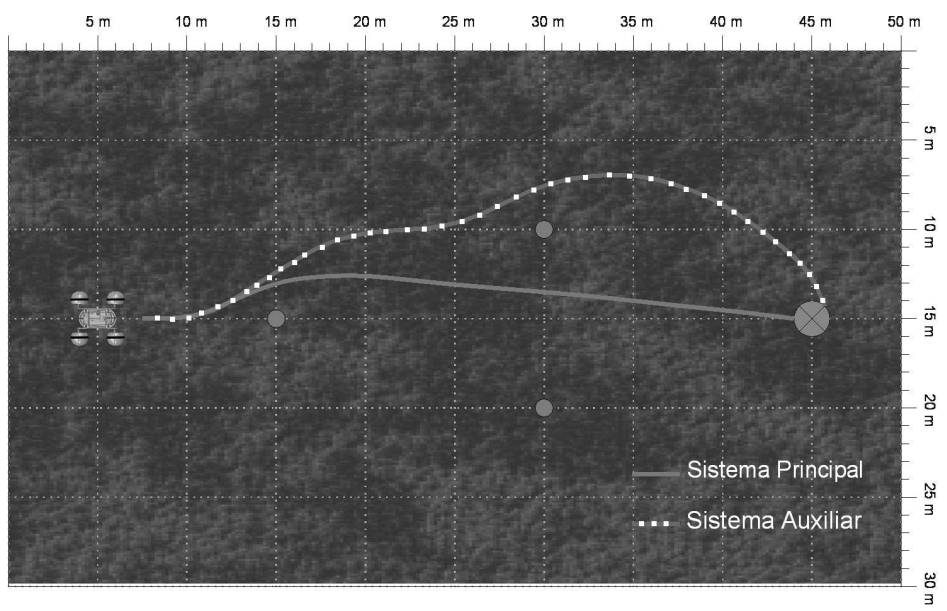

Figura 13: Experimento 1.

- Experimento 2 (Figura 14): A distribuição de obstáculos foi idealizada de maneira a forçar um trajeto pelo lado esquerdo. Novamente, pode se verificar a diferença de eficiência entre os sistemas, embora o SAN tenha atingido o objetivo.

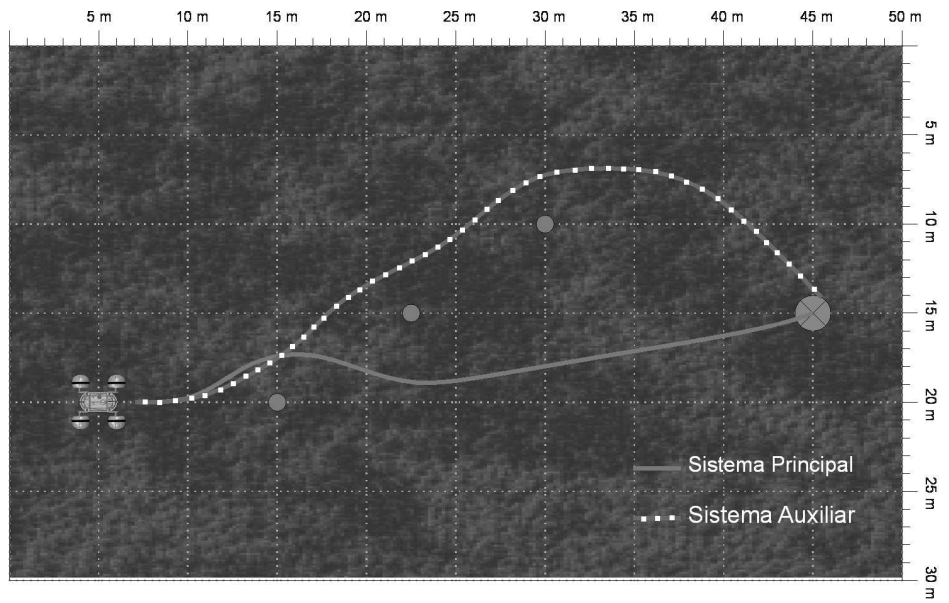

Figura 14: Experimento 2. 
- Experimento 3 (Figura 15): Este é um espelho do experimento 2; a configuração dos obstáculos induz a uma trajetória ótima pelo lado direito. Os resultados foram similares aos obtidos no experimento 2: o SPN foi mais eficiente que o SAN, mas este conseguiu novamente atingir o objetivo.

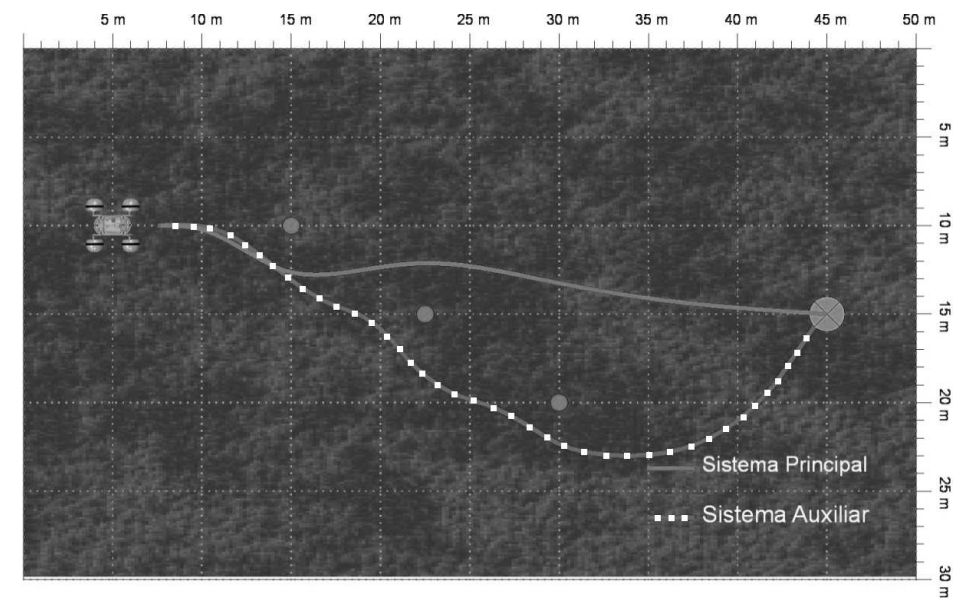

Figura 15: Experimento 3.

- Experimento 4 (Figura 16): Similar ao experimento 1, não indicando um lado preferencial para desvio dos obstáculos. A sofisticação dos equipamentos permitiu ao SPN uma maior eficiência; os equipamentos do SAN, por exemplo, não permitem detectar obstáculos a uma distância maior do que cinco metros. Assim, o robô controlado pelo SAN só consegue se esquivar e construir uma rota alternativa até o objetivo após detectar a presença dos obstáculos.

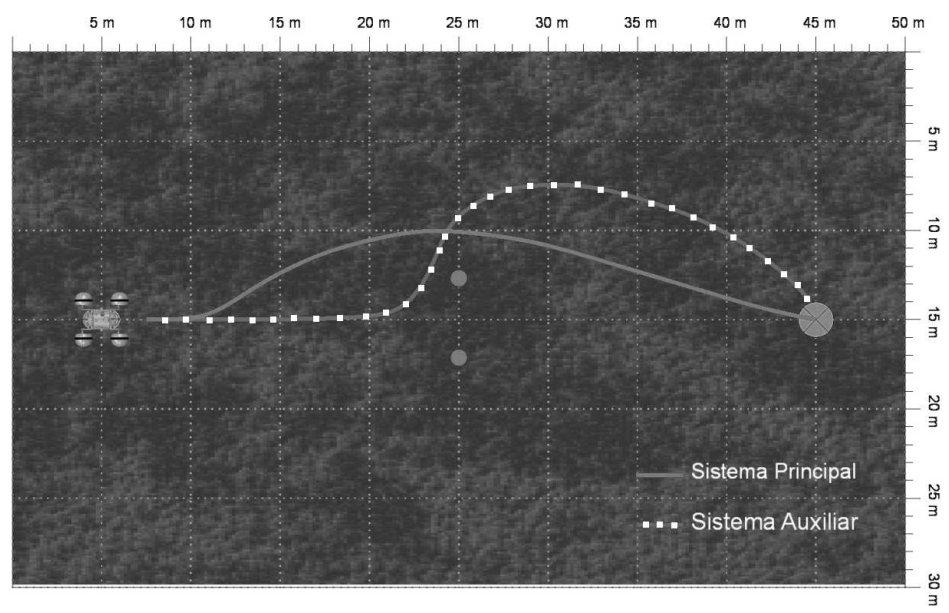

Figura 16: Experimento 4. 
- Experimento 5 (Figura 17): O objetivo era forçar o robô a girar para a direita e se deslocar rumo ao objetivo. Observa-se que o SPN consegue construir uma melhor rota, ao passo que o SAN (linha pontilhada), devido à limitação de 5 metros para a distância de aquisição de dados, não consegue detectar os outros obstáculos e segue pela esquerda. Conforme os detecta, procura se esquivar e prosseguir rumo ao objetivo.

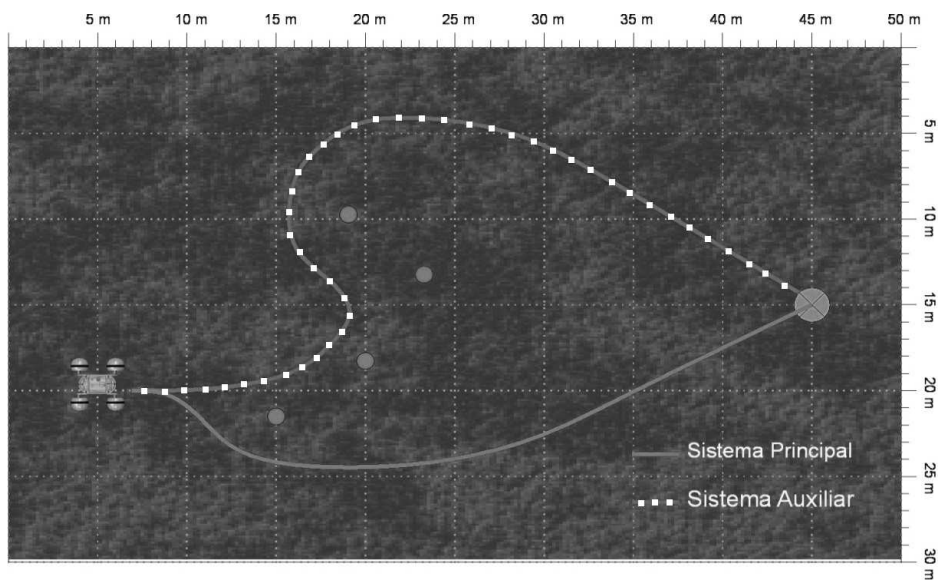

Figura 17: Experimento 5.

- Experimento 6 (Figura 18): Nesta distribuição aleatória de obstáculos, o comportamento do SAN foi bastante similar ao do SPN, apenas um pouco menos suave nas proximidades do obstáculo.

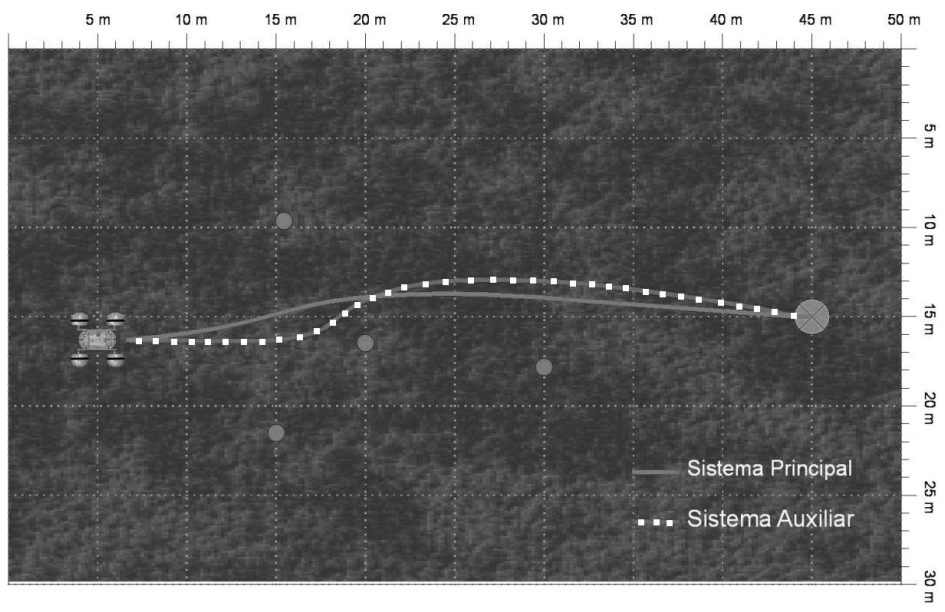

Figura 18: Experimento 6. 
- Experimento 7 (Figura 19): Quatro obstáculos foram dispostos de modo a formar um corredor estreito que permitiria ao robô atingir o objetivo sem necessidade de se esquivar de obstáculo algum. Conforme apresentado na Figura 19, o SPN produz uma trajetória praticamente retilínea, enquanto que, quando controlado pelo SAN, o robô tende a se esquivar dos obstáculos detectados nas zonas laterais, perdendo um pouco de eficiência no trajeto realizado.

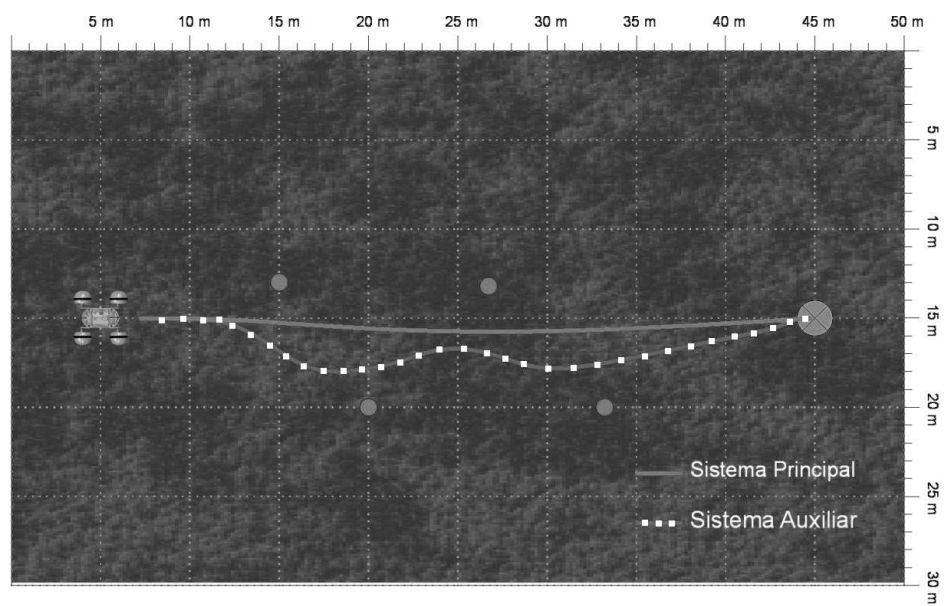

Figura 19: Experimento 7.

- Experimento 8 (Figura 20): Esta situação, mostrada na Figura 20, foi a única em que o robô controlado pelo SAN não conseguiu atingir o objetivo. A distribuição dos obstáculos faz com que o robô os detecte já muito próximo do objetivo, fazendo com que sempre se desvie de um deles e acabe por não atingir o objetivo.

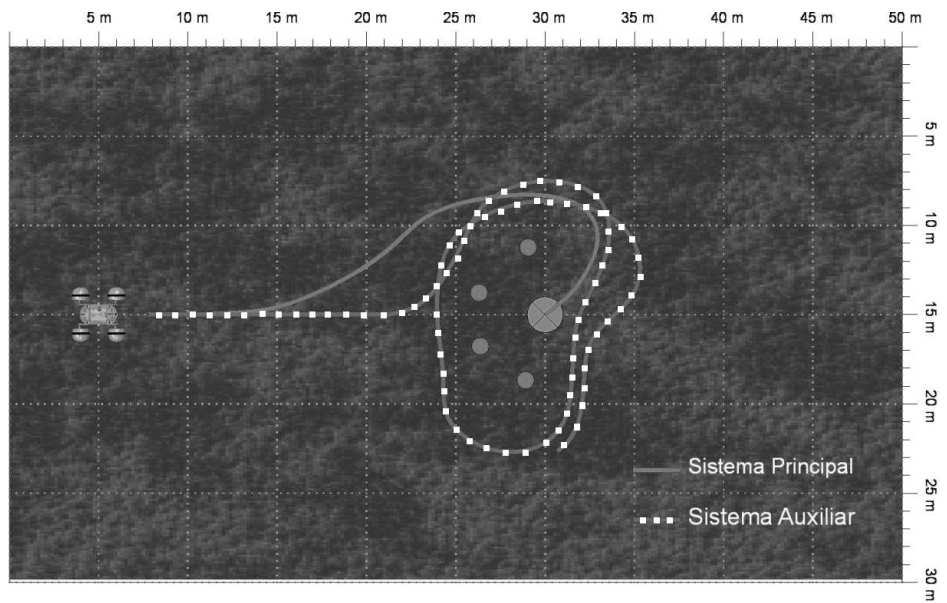

Figura 20: Experimento 8. 
- Experimento 9 (Figura 21): Os quatro obstáculos formam um retângulo e a distância entre os dois primeiros é insuficiente para o robô passar entre eles. Os resultados obtidos foram bons tanto para o SAN como para o SPN, uma vez que em nenhum dos casos o RAHM colidiu com os obstáculos. A diferença está na eficiência das trajetórias.

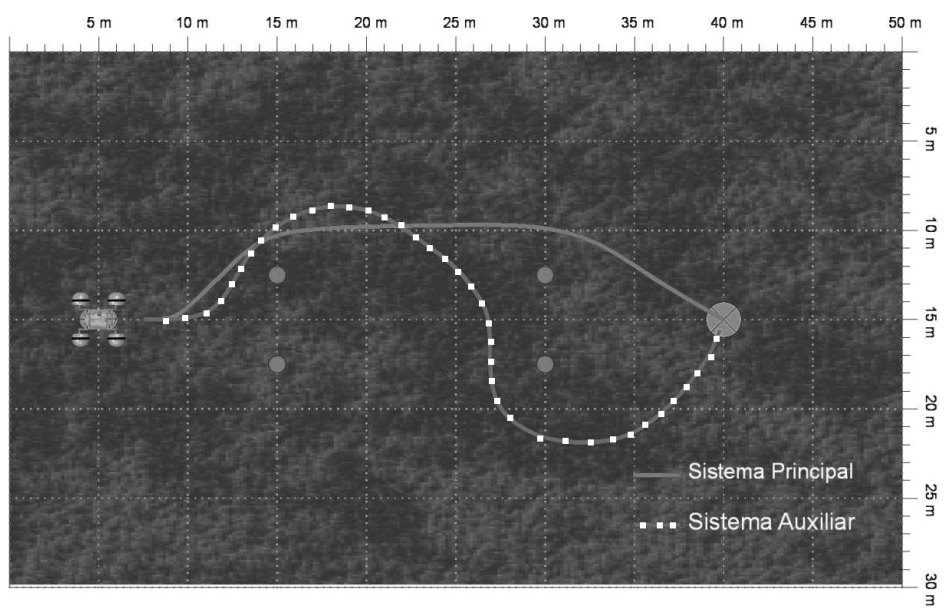

Figura 21: Experimento 9.

- Experimento 10 (Figura 22):

Objetivo similar ao do experimento 6 , com obstáculos distribuídos aleatoriamente. Os resultados foram similares aos do experimento 6 , inclusive no que tange à eficiência.

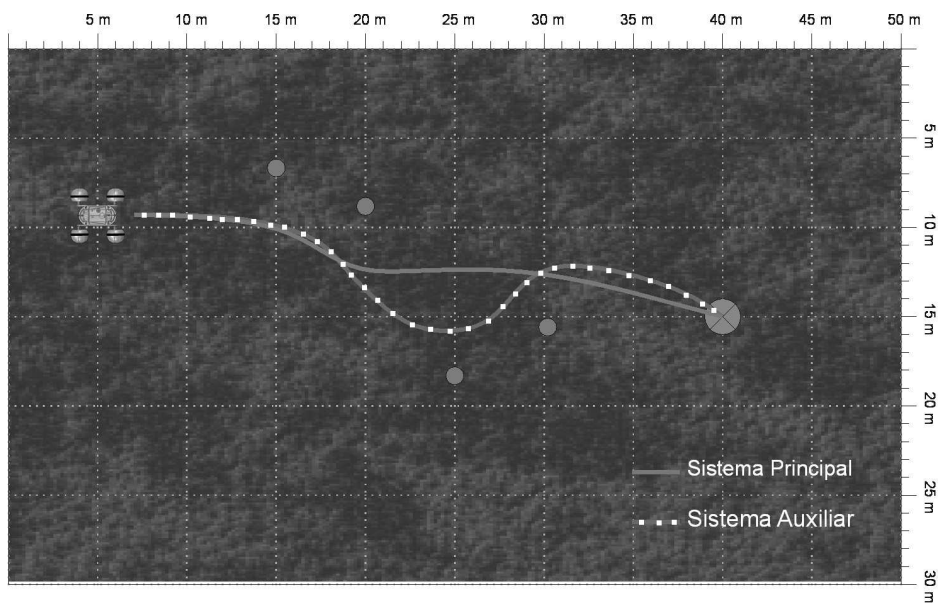

Figura 22: Experimento 10. 
É importante ressaltar a diferença existente entre os dois sistemas no tocante aos sensores: o sensor laser, equipamento essencial no SPN, consegue mapear todo o ambiente dentro de um raio de 80 metros à frente, permitindo ao robô construir a rota mais eficiente, já que o ambiente é de apenas 50 metros; o SAN, por outro lado, necessita construir a rota em função das informações obtidas de uma visão máxima de 5 metros. Mesmo com esta desvantagem, o SAN apresentou bons resultados, com um custo financeiro e computacional bastante menor e com um consumo energético baixo.

\subsection{Comparação dos Sistemas de Navegação}

Fazendo uso das métricas mencionadas anteriormente (consumo de energia, custo financeiro, custo computacional e simplicidade), a Tabela 1 apresenta os resultados comparativos para cada sistema de navegação.

No caso do SAN, é monitorada apenas a memória do PC remoto, identificado na tabela com a sigla CR; no caso do SPN, monitoram-se tanto a memória do PC embarcado (CE) como a do PC remoto (CR). Percebe-se que o SAN não satura a placa de rede, pois faz uso de apenas $15 \%$ do seu poder. Além disso, a memória do PC remoto é utilizada em $50 \%$, já levando em conta o custo computacional da execução do controlador fuzzy programado em Visual Studio. O consumo de corrente do sistema é de 6,5 Amperes em média. No caso do SPN, observa-se um maior consumo de corrente, tempos mais curtos para realizar os diferentes experimentos e custo computacional mais alto.

Destacam-se, na Tabela 1, os itens com melhor resultado para os sistemas utilizados. Percebe-se que o SAN é um sistema menos eficiente em relação ao tempo de percurso, mas necessita de apenas um pouco mais da metade da energia requerida pelo SPN.

Os dez experimentos, além de servirem para se avaliar o sistema auxiliar de navegação, foram importantes para testar o RAHM de forma geral, depurar todos os sistemas e identificar eventuais problemas específicos. Foi encontrado, por exemplo, um problema mecânico - roda dianteira direita mecanicamente freada - que ocasionava um maior consumo de corrente quando o robô girava para a direita.

\section{CONCLUSÕES}

Este trabalho apresentou o desenvolvimento de um controle para o Sistema Auxiliar de Navegação (SAN) de um robô ambiental híbrido denominado RAHM. O sistema resultante deveria atender a imposições do projeto: baixo consumo de energia, baixo custo financeiro e computacional, simplicidade e desempenho satisfatório quando comparado ao do Sistema de Navegação Primário, que conta com equipamentos mais caros e sofisticados.

O controle do SAN foi realizado por meio de um Sistema Fuzzy Hierárquico e as expectativas de um desempenho satisfatório foram confirmadas para um conjunto de dez experimentos: em nove 
Tabela 1: Comparação entre o SAN e o SPN.

\begin{tabular}{|c|c|c|c|c|c|c|c|c|c|}
\hline \multicolumn{2}{|c|}{ Tempo (Seg) } & \multicolumn{2}{|c|}{$\begin{array}{l}\text { Consumo de } \\
\text { corrente }(\mathrm{mA})\end{array}$} & \multicolumn{2}{|c|}{$\begin{array}{c}\text { Corrente X } \\
\text { tempo }(\mathrm{mA} / \mathrm{h})\end{array}$} & \multicolumn{2}{|c|}{$\begin{array}{c}\text { Custo } \\
\text { Computacional }(\%)\end{array}$} & \multicolumn{2}{|c|}{$\begin{array}{c}\text { Dados } \\
\text { na Rede }(\%)\end{array}$} \\
\hline SAN & SPN & SAN & SPN & SAN & SPN & SAN & SPN & SAN & SPN \\
\hline $1: 43$ & $1: 32$ & 6468 & 10415 & 185,05 & 266,16 & $51 \%$ CR & $\begin{array}{l}76 \% \mathrm{CE} \\
65 \% \mathrm{CR}\end{array}$ & $12,8 \%$ & $76 \%$ \\
\hline 2:04 & $1: 30$ & 6820 & 10115 & 234,91 & 252,87 & $48 \%$ CR & $\begin{array}{l}81 \% \mathrm{CE} \\
67 \% \mathrm{CR}\end{array}$ & $12,5 \%$ & $82 \%$ \\
\hline $2: 07$ & $1: 28$ & 7430 & 10815 & 262,11 & 225,31 & 49\% CR & $\begin{array}{l}80 \% \mathrm{CE} \\
67 \% \mathrm{CR}\end{array}$ & $13 \%$ & $82 \%$ \\
\hline $1: 27$ & $1: 15$ & 7115 & 10085 & 171,94 & 210,1 & $52 \% \mathrm{CR}$ & $\begin{array}{l}78 \% \mathrm{CE} \\
65 \% \mathrm{CR}\end{array}$ & $11,6 \%$ & $86 \%$ \\
\hline $2: 10$ & $1: 10$ & 6680 & 10225 & 241,22 & 198,81 & $51 \% \mathrm{CR}$ & $\begin{array}{l}90 \% \mathrm{CE} \\
64 \% \mathrm{CR}\end{array}$ & $14,3 \%$ & $78 \%$ \\
\hline 1:03 & 55 & 6955 & 10180 & 121,71 & 155,52 & $51 \% \mathrm{CR}$ & $\begin{array}{l}83 \% \mathrm{CE} \\
63 \% \mathrm{CR}\end{array}$ & $12,8 \%$ & $80 \%$ \\
\hline 1:08 & 1:00 & 6200 & 9980 & 117,11 & 166,33 & $46 \% \mathrm{CR}$ & $\begin{array}{l}77 \% \mathrm{CE} \\
66 \% \mathrm{CR}\end{array}$ & $11,9 \%$ & $85 \%$ \\
\hline & 52 & - & 10345 & . & 149,42 & & $\begin{array}{l}86 \% \mathrm{CE} \\
73 \% \mathrm{CR}\end{array}$ & - & $82 \%$ \\
\hline $1: 35$ & $1: 12$ & 7050 & 10280 & 186,04 & 205,6 & $50 \%$ CR & $\begin{array}{l}82 \% \mathrm{CE} \\
64 \% \mathrm{CR}\end{array}$ & $12,7 \%$ & $78 \%$ \\
\hline $1: 48$ & $1: 10$ & 6650 & 9720 & 199,5 & 189 & $47 \% \mathrm{CR}$ & $\begin{array}{l}84 \% \mathrm{CE} \\
64 \% \mathrm{CR}\end{array}$ & $12,5 \%$ & $75 \%$ \\
\hline
\end{tabular}

experimentos, o SAN conseguiu atingir o objetivo, mostrando-se confiável frente às limitações de equipamento e às características desejadas.

Por não ser dotado de sensor a laser - presente apenas no SPN -, que permite o mapeamento de todo o ambiente, o SAN mostrou-se, em geral, menos eficiente do que SPN em termos de trajetórias da origem ao objetivo especificado. Por outro lado, o SAN apresenta custos bastante inferiores ao do SPN, configurando-se em uma excelente alternativa para situações de emergência.

Como trabalhos futuros, pode-se destacar a possível utilização de um método de sintonia por aprendizado para adaptação do SIF a eventuais mudanças de características do robô, como, por exemplo, peso ou atrito nas rodas, bem como possibilitar a configuração de parâmetros do SIF na interface gráfica, pois a atual não apresenta esta funcionalidade. 


\begin{abstract}
This paper presents the development of a fuzzy logic-based auxiliary navigation system for a Medium-Sized Hybrid Environmental Robot. The electronic project and the navigation system programming are also presented. The system's behaviour is evaluated against the robot's main navigation system.
\end{abstract}

Keywords: Fuzzy logic; Autonomous robotics; Navigation; Control.

\title{
REFERÊNCIAS
}

[1] "Visual Studio. Development Application". https://www.visualstudio.com/support.

[2] A. Barral Silva. "Modelagem de Sistemas Robóticos Móveis para Controle de Tração em Terrenos Acidentados". Master's thesis, PUC-Rio (2007).

[3] G. Cook. "Mobile Robots: Navigaton, Control and Remote Sensing". Wiley-IEEE Press (2011).

[4] J.P. Cordoba, C.A. Restrepo \& L.H. Ríos. Descriptión de una Plataforma Móvil Implementada Para El Estudio de Técnicas Inteligentes de navegación: P-metin. Scientia et Technica, XI(27) (2005), 37-42.

[5] C. Gómez. "CONTROLE FUZZY DE UM SISTEMA AUXILIAR de Navegação de um Robô Ambiental Híbrido”. Master's thesis, PUC-Rio (2016).

[6] F. Gomide, R.R. Gudwin \& R. Tanscheit. Conceitos fundamentais da teoria de conjuntos fuzzy, lógica fuzzy e aplicações. In "6th IFSA Congress: Tutorials" (1995), pp. 1-38.

[7] C.K. Lee, C.L. Lin \& B.M. Shiu. Autonomous vehicle parking using artificial intelligent approach. In "4th International Conference on Autonomous Robots and Agents" (2000), pp. 496-451.

[8] J.M. Mendel. Fuzzy logic systems for engineering: a tutorial. Proceedings of the IEEE, 83(3) (1995), $345-377$.

[9] C. Rodrigo \& A. Cipriano. Sistema de Guiado para un robot móvil basado en lógica difusa. In "XV Congreso de la Asociación Chilena de Control Automático" (2002), pp. 387-392.

[10] A. Saffiotti. The Uses of Fuzzy Logic in Autonomous Robot Navigation. Soft Computing, 1(4) (1997), 180-197.

[11] A. Saffiotti. Fuzzy logic in autonomous navigation. In D. Driankov \& A. Saffiotti (editors), "Fuzzy Logic Techniques for Autonomous Vehicle Navigation". Springer-Physica Verlag (2001), pp. 3-24.

[12] P. Stein \& N. Reis. Sistema integrado de navegação para o Robô Ambiental Hibrido na Floresta Amazônica. In “XIII Simpósio Brasileiro de Sensoriamento Remoto” (2007), pp. 21-26.

[13] K. Tanaka. "An introduction to fuzzy logic for practical applications". Springer (1996).

[14] C. von Altrock. "Fuzzy logic and Neuro Fuzzy applications in business and finance". Prentice Hall (1996).

[15] B.F. Wu, T.T. Lee, H.H. Chang, J. J-J, C.N. Lien, T.Y. Liao \& J.W. Perng. GPS navigation based autonomous driving system design for intelligent vehicles. In "IEEE International Conference on Systems, Man and Cybernetics” (2007), pp. 3294-3299.

[16] J. Yen, R. Langari \& L. Zadeh. "Industrial applications of fuzzy logic and intelligent systems". IEEE Press (1994). 